\title{
IR Sensor Based on Low Bandgap Organic Photodiode With Up- Converting Phosphor
}

Kettle, J.P.; Kettle, J.; Chang, S.W.; Horie, M.

\section{IEEE Sensors Journal}

DOI:

10.1109/JSEN.2015.2394744

Published: 28/01/2015

Peer reviewed version

Cyswllt i'r cyhoeddiad / Link to publication

Dyfyniad o'r fersiwn a gyhoeddwyd / Citation for published version (APA):

Kettle, J. P., Kettle, J., Chang, S. W., \& Horie, M. (2015). IR Sensor Based on Low Bandgap Organic Photodiode With Up-Converting Phosphor. IEEE Sensors Journal, 15(6), 3221 - 3224. https://doi.org/10.1109/JSEN.2015.2394744

\section{Hawliau Cyffredinol / General rights}

Copyright and moral rights for the publications made accessible in the public portal are retained by the authors and/or other copyright owners and it is a condition of accessing publications that users recognise and abide by the legal requirements associated with these rights. study or research.

download and print one copy of any publication from the public portal for the purpose of private

- You may not further distribute the material or use it for any profit-making activity or commercial gain

- You may freely distribute the URL identifying the publication in the public portal ?

\section{Take down policy}

(c) 2015 IEEE. Personal use of this material is permitted. Permission from IEEE must be obtained for all other users, including reprinting/ republishing this material for advertising or promotional purposes, creating new collective works for resale or redistribution to servers or lists, or reuse of any copyrighted components of this work in other works.

Take down policy

If you believe that this document breaches copyright please contact us providing details, and we will remove access to the work immediately and investigate your claim. 
IR Sensor based on low bandgap organic photodiode with up-converting phosphor

\section{J. Kettle, S-W. Chang, M. Horie}

Abstract-We report an InfraRed (IR) sensor which is fabricated by integrating a PCPDTBT:PCBM Organic PhotoDiode (OPD) with an Up-Converting (UC) phosphor. The UC phosphor extends the response range by absorbing incoming light with a wavelength of $986 \mathrm{~nm}$ and re-emitting at $804 \mathrm{~nm}$, which is a wavelength that can be absorbed by the active layer, resulting in a generation of a photocurrent. In order to ensure low reverse bias leakage current, PEDOT:PSS was not used as a hole transporting layer, which reduced reverse leakage current by two orders of magnitude compared to conventional hole transporting layers. An IR-emitting laser diode (with emission at $986 \mathrm{~nm}$ ) is used as a light source to illuminate the sensor. The results demonstrate the proof of principle of sensing using polymer-based OPDs in the NearInfrared, with wider applications possible in areas such as telecommunications or sensors if different upconverting phosphors are applied.

Index Terms - Infrared imaging, Optical sensors, Optical detectors, Organic semiconductors

\section{INTRODUCTION}

Infrared (IR) photodetectors have attracted significant recent interest owing to of their broad range of

This paragraph of the first footnote will contain the date on which you submitted your paper for review. This work was supported in part by Welsh Crucible under the small grant scheme, "ARMED".

J. Kettle is based at the School of Electronics, Bangor University, Dean St., Bangor, Gwynedd, Wales, UK (email: j.kettle@bangor.ac.uk).

M. Horie and S-W. Chang are based at Department of Chemical Engineering, National Tsing Hua University, 101, Sec. 2, Kuang-Fu Road, Hsinchu, Hsinchu city, Taiwan 30013, ROC. applications in modern technology, such as telecommunications, spectroscopy, medical diagnosis and for sensing applications such as for detection of Volatile Organic Compounds (VOCs), Carbon Dioxide $\left(\mathrm{CO}_{2}\right)$ or methane $[1,2,3]$. Whilst there are a number of different types of commercially available IR sensors, most IR photodiodes are based on inorganic semiconductors such as Gallium Indium Arsenide (GalnAs), Indium Arsenide (InAs) Quantum dots or Germanium (Ge) [4]. Whilst the performance in terms of spectral sensitivity of these material systems is very high, photodiodes could be improved by taking advantage of many of the benefits seen in the emerging field of organic electronics.

Organic Photodiodes (OPDs) could have a number of advantages over the incumbent technology, including low cost, solution processability and flexibility, which could enable IR photodiodes to be placed onto non-flat surfaces $[5,6]$. With the fast development of organic electronics in the past decade, the carrier mobilities in some organic semiconductors are comparable to or even higher than that of amorphous silicon, implying their broad potential IR sensing applications in the future [7]. OPDs possess high on/off ratio of photocurrent during illumination when compared to the dark current (typically $>3$ orders of magnitudes). Another major advantage is that the absorption profile can be 'tuned' to absorb most visible wavelengths by altering the semiconductor inside the active layer within the active layer. Currently, few demonstrations exist with polymer-based OPDs operating in the NIR region. Some narrow-bandgap evaporated organic semiconductors have been used in IR photodetectors [8]. Composite films of QDs and organic semiconductors have also been used in IR sensors, however, the responsivity of the device to IR light illumination is very low and there is low selectivity to visible radiation mentioned [9].

An alternative approach is to use an UpConversion (UC) layer to 'up-shift' IR radiation into the part of the visible spectrum where organic polymers absorb. In this paper, we report the integration of a luminescent UC phosphor with a PCPDTBT:PCBM organic photodiode for applications in IR detection. This 


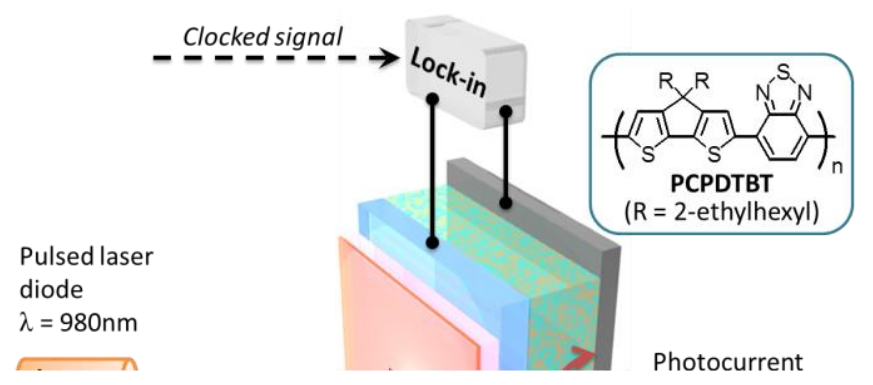

Fig. 1. Experimental setup for the IR sensor; enables a polymer based OPD to extend the operation in the NIR region, where few examples currently have been reported. One of the main advantages in this approach is using an organic sensing layer allows the active layer material to be selected that matches the emission spectrum of the UC-layer, due to the vast arrays of active layer materials currently available. Whilst the phosphor selected, absorbs around $986 \mathrm{~nm}$, other UC material could be used to extend the IR absorption to around $2000 \mathrm{~nm}$.

Experimental

The image in Fig. 1 provides a schematic representation of the device structure and experimental setup used to demonstrate the proof of principle for extended IR sensing. Initially an ITO coated glass is prepared by cleaning. Two different device architectures were trialed. For a control device, PEDOT:PSS, was used as the Hole Transport Layer (HTL), which was applied by spin coating at $5000 \mathrm{rpm}$ for 30 seconds and subsequently baked at $120^{\circ} \mathrm{C}$ for 20 minutes. Otherwise, devices were fabricated without PEDOT:PSS. Clevios ${ }^{\mathrm{TM}}$ PEDOT:PSS(P VP Al 4083) was purchased from Hereaus $\mathrm{GmbH}$., Germany. For this work, we used poly[4,4-bis(2ethylhexyl)-4H-cyclopenta[2,1- $\left.b ; 3,4-b^{\prime}\right]$ dithiophene-2,6diyl-alt-2,1,3-benzothiadiazole-4,7-diyl] (PCPDTBT) (the synthesis and performance is reported in [10]) and $\mathrm{C}_{71^{-}}$ PCBM (Nano-C) blended in chlorobenzene at $30 \mathrm{mg} / \mathrm{mL}$ with $75 \% \mathrm{C}_{71}$-PCBM content. A processing additive, 1,8- octanedithiol was added to the solution. The active layer was annealed at $80^{\circ} \mathrm{C}$ for 2 hours after coating. After coating the active layer, the Calcium and Aluminium cathode was thermally evaporate ed onto the through shadow masks at a pressure of $\sim 1 \times 10^{-6}$ mbar at rates of $\sim 5 \AA / s$ to define an active area of $0.2 \mathrm{~cm}^{2}$. Finally, a coating of an Up-Converting (UC) phosphor dissolved in butyl acetate was used was applied to the air side of the OPD. The UC material was supplied by Phosphor Technology Ltd., UK (PTIR 660). This exhibits peak absorption at $975 \mathrm{~nm}$ with reemission occurring in two wavelength regions; firstly between $790 \mathrm{~nm}$ and $810 \mathrm{~nm}$ (peak emission occurs at $804 \mathrm{~nm}$ ) and also $640 \mathrm{~nm}$ to $700 \mathrm{~nm}$ (three peaks are present in this range at $661 \mathrm{~nm}, 676 \mathrm{~nm}$ and $683 \mathrm{~nm}$ ). To maximize the light capture, a relatively low band gap organic active layer is necessary for absorption of all reemitted light; for this reason, PCPDTBT was selected. The cells were then encapsulated and tested.

Electrical testing of the cells was undertaken using a Stanford Research system (SR810) lock-in amplifier with a DC bias varied between 0 and $-5 \mathrm{~V}$ across the device. External Quantum Efficiency (EQE) properties of the OPD are reported elsewhere [10], but current is generated in all wavelengths between $320-850 \mathrm{~nm}$; including the UC phosphor extends the response to $986 \mathrm{~nm}$. For illumination, a laser diode was purchased from Thorlabs and focused using initially using power meter. The Laser diode device was DC biased using a Keithley 2600 SMU and the optical signal was modulated using an chopper in order to improve the signal-to-noise ratio and was synchronised with the lock-in amplifier at $60 \mathrm{~Hz}$.

Responsivity measurements were made by illuminating the top surface of the OPD at normal incidence, The OPD response was measured in photoconductive mode, so an external reverse bias is applied via a Keithley 2600 SMU and the current was measured using a lock-in amplifier. Measurements were undertaken in ambient laboratory conditions.

results

Electrical response 
Initially the OPD was tested for sensitivity and functionality. One potential issue with the setup is that OPDs are not well suited for weak light detection because they suffer from high dark-current up to $1 \mathrm{~mA}$ $\mathrm{cm}^{-2}$ under reverse bias, which is exasperated due to the relatively low shunt resistance seen in PCPDTBT:PCBM OPDs. Therefore, in order to increase the dynamic range of the OPD, the on/off ratio has to be enhanced by reducing the dark current. Other reports to minimize dark current include using crosslinkable HTL's [11] or approaches similar to discussed below, where the HTL is completely removed [12].

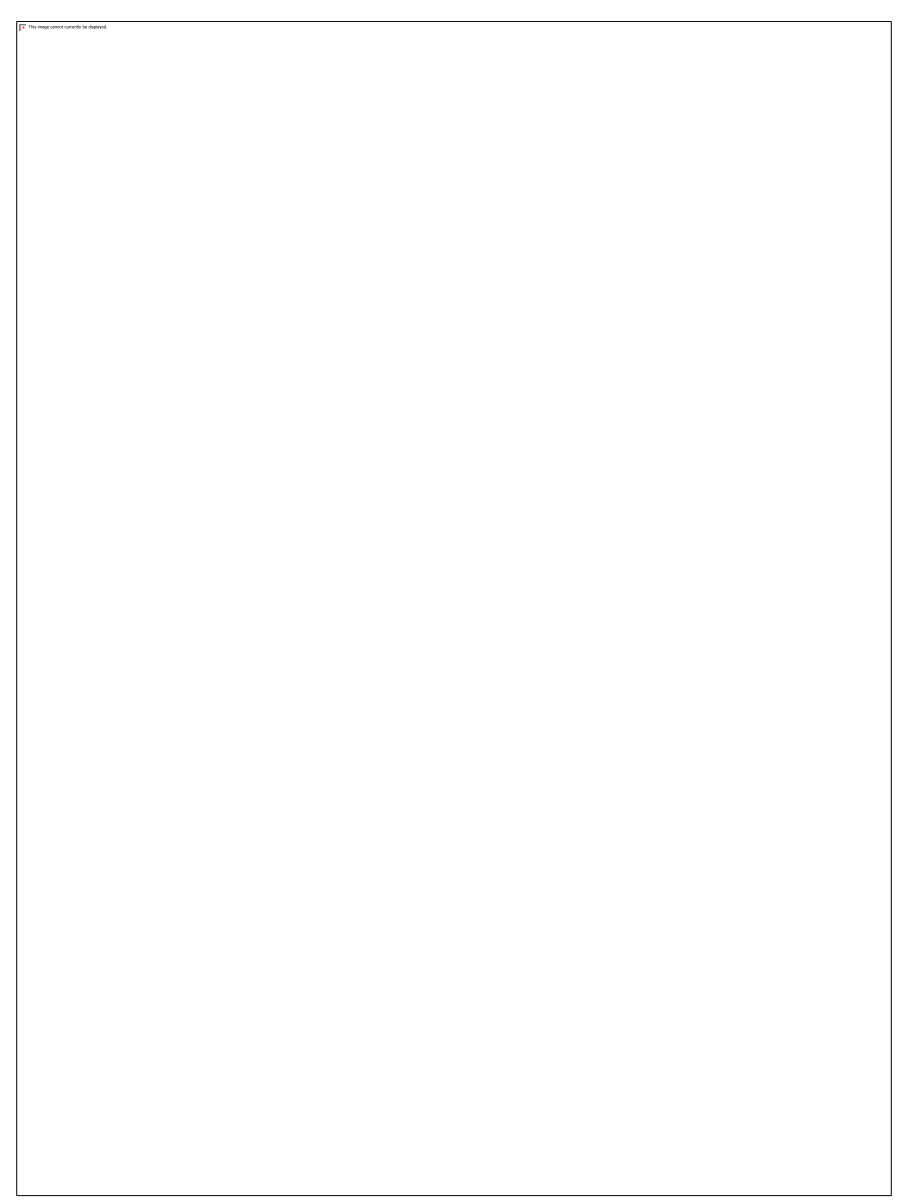

Fig.2. (a) Dark current of the Organic Photodiode using a PEDOT:PSS HTL and without PEDOT:PSS, showing low leakage under reverse bias and the relative reduction in dark current by removing PEDOT:PSS and (b) The reduction in dark current versus applied voltage
Figure 2 shows the dark current for devices for OPDs based on two different device configurations; with and without a PEDOT:PSS HTL. Ordinarily in organic devices (Such as Organic Light Emitting Diodes, LED or PhotoVoltaics, OPVs), a Hole Injection Layer or HTL is inserted between the transparent anode and active layer. The main function of this is to ensure a better match in energies between the LUMO of the polymer and work function of the anode, enabling efficient charge injection (for OLEDs) or extraction (for OPVs) under forward bias. PEDOT:PSS is one of the most commonly reported HTLS and possesses a number of good characteristics for OPD devices; it has high conductivity, the HOMO energy level has a $\sim 0.4 \mathrm{eV}$ energy difference with the HOMO energy level of PCPDTBT and once it is coated, PEDOT:PSS provides a planarised interface between the anode and the active region $[13,14,15]$. However, OPDs are operated in reverse bias, so ensuring a matching work function between the anode and PCPDTBT:PCBM layer is not as important. In addition, the data shown in in Figure 2, the dark current under reverse bias is significant $\left(\sim 0.5 \mathrm{~mA} / \mathrm{cm}^{2}\right.$ at $\left.\mathrm{V}_{\mathrm{BIAS}}=-2 \mathrm{~V}\right)$. Therefore, by using PEDOT:PSS as the hole transport layer, the OPD sensor will be limited by the high noise level of the device, imposing a restriction on the minimum optical power that can be detected. Minimization of dark current in the setup shown in Fig. 1 is imperative, as this limits the minimum detectable power. This is exasperated as the up-conversion efficiency of the UC phosphor is low $(<5 \%)$, so the output from the UC layer will also be low (even under high lasing powers) and would only be detectable if the dark current is low enough to distinguish the photocurrent from noise, so for the following experiments OPDs without PEDOT:PSS were used, which demonstrates over two orders of magnitude reduction in dark current.

\section{Optical response}

Figure 3 shows the response of the OPD to varying drive current of the laser diode, obtained by incrementing the drive current of the IR laser diode. Overall, the DC response is low compared to silicon or germanium photodiodes and is limited by the low 
quantum efficiency of UC layer. The IR laser was DC biased initially with $0 \mathrm{~mA}$ drive and increased in steps until the laser diode threshold current was reached $(\sim 37 \mathrm{~mA})$. At a drive current just over the threshold current, the OPD response increases at an approximately linear rate and as the drive current through the Laser diode increased. At $52 \mathrm{~mA}$, the Laser diode begins to saturate as a result of Gain saturation in the active region, leading to a similar non-linear response from the OPD.

The responsivity of a photodiode is defined as a ratio of output photocurrent $\left(\mathrm{I}_{\mathrm{PD}}\right)$ from the OPD to the incident light power (P) from the laser diode, or $\mathrm{x}$. Power output from the laser diode was calibrated by using an optical power meter. Shown also in Fig 3 is the responsivity as a function drive current from the Laser diode. No photocurrent is measured by the OPD below the threshold current of the laser diode When comparing to other commercially available photodiodes, the responsivity is comparatively low; this is limited also by the low conversion efficiency of the UC material. As input power is increased, a relatively small decrease in $R(\lambda)$ is observed; approximately a $20 \%$ decrease over the measured range (this corresponds to 150x change in magnitude of input power from the IR laser diode. Since the photodiode current generated by the PCPDTBT:PCBM active layer is proportional on the number of electron-hole (e-h) pairs, this variation in responsivity is likely to arise from a power dependence of the UC mechanism. Power dependence of UC layers has been reported by others $[16,17]$ and varies as a result of different responses from up-converting transitions as laser power is varied. However, the sublinear saturation, which occurs at drive current $>52 \mathrm{~mA}$ is primarily due to heating of the laser diode, as the device was not actively cooled. If the setup shown in Fig, 1 could integrate a thermoelectric cooling element, then a linear OPD response up to $\sim 100 \mathrm{~mA}$ drive current would be observed.

Assuming that the shot noise current associated to the dark current is dominant over the thermal noise, the Noise Equivalent Power (NEP, in $\mathrm{W} / \mathrm{Hz}^{1 / 2}$ ) can be expressed as $N E P=I_{D} / R(\lambda)$, where $I_{D}$ is the OPD dark current. The NEP obtained for our photodetector is
$1.23 \times 10^{-11} \mathrm{~W} / \mathrm{Hz}^{1 / 2}$, indicating that low levels of light can be used in this configuration. In addition, the specific detectivity $\left(D^{*}\right)$, that indicates the ability to detect low levels of incident power, is given by $D^{*}=\sqrt{A} / N E P$, where $A$ is device area. We calculate a $D^{*}$ of $3.64 \times 10^{8}$ Jones. Whilst these values of NEP and $D^{*}$ are in the slightly lower than values obtained by other authors for OPDs, it is limited by the low conversion efficiency of the UC material.

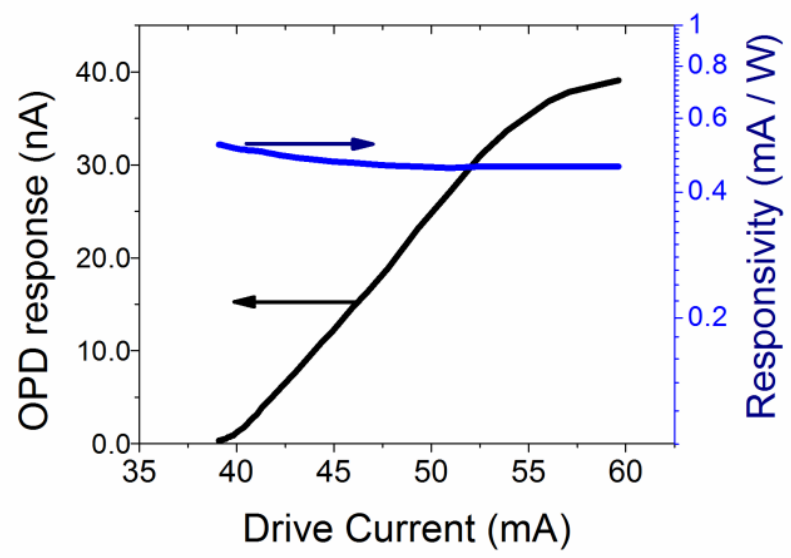

Fig.3. The output current from the OPD as a function of drive current from the IR Laser. The OPD was biased at $-2 \mathrm{~V}$. Overlaid on the graph is the OPD responsivity as a function of input power from the IR laser

Frequency response

Finally the frequency response of the OPD is studied by varying the chopped frequency of the incoming light. Figure 4 shows the normalized frequency response of the OPD at $0 \mathrm{~V}$, $-2 \mathrm{~V}$ and $-4 \mathrm{~V}$ under laser illumination. Measurements carried out with the lock-in amplifier (which is limited up to $100 \mathrm{kHz}$ ). At $0 \mathrm{~V}$ bias, the $-3 \mathrm{~dB}$ cut off frequency is measured at 4.40 $\mathrm{kHz}$. The photodetector bandwidth increases slightly with reverse bias reaching up to $6.3 \mathrm{kHz}$ at $-4 \mathrm{~V}$. These values are similar to those measured for OPD photodetectors $[6,7]$, when considering the lower light output from the UC layer and this speed response is good enough for many instrumentation applications. 


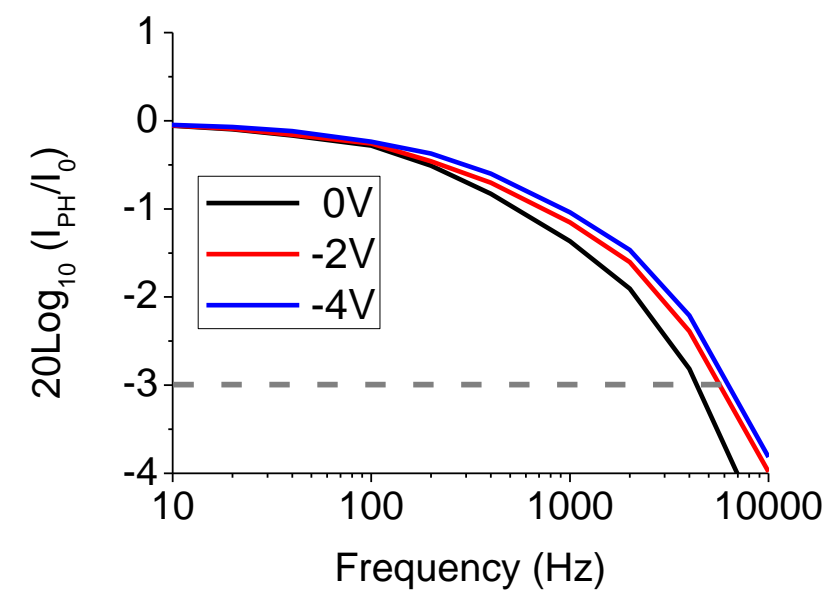

Fig.4. Amplitude bode plot for OPD under three bias conditions $\left(V_{B I A S}=O V\right.$, $2 \mathrm{~V},-4 \mathrm{~V}$ ) with wavelength illumination at $986 \mathrm{~nm}$.

\section{Conclusion}

In conclusion, we have demonstrated the fabrication, and characterization of an IR sensor using a polymerbased Organic Photodetector with an optical UC phosphor layer. The use of the UC layer further extends the absorption range of the OPD into the NIR. To measure the response of the UC layers, devices had to be constructed without a PEDOT:PSS HTL, which is shown to reduce the dark current by over two orders of magnitude. The data presented in this report indicates that there is significant future potential if further optimisation is undertaken of the UC materials, in particular if the low quantum efficiency can be improved. It is known that OPDs that operate in the visible spectrum have optical response close to inorganic light sensors. By improving the UC materials, the responsivity could potentially be increased to a level comparable to IR extended photodiodes, CCDs and CMOS sensors. The results demonstrate the proof of principle of sensing using polymer based OPDs in the Near-Infrared, with wider applications possible in areas such as telecommunications, imaging, light and portable sensors, if suitable up-converting phosphors are sourced. However, for high speed applications, one further limitation might the frequency response. Future work should not just focus on improving the efficiency, but also the operational speed of these devices

Acknowledgment
The Authors would like to thank the Welsh Crucible team for supporting this work and in particularly $\mathrm{Dr}$ Vincent Teng (Swansea University) and Dr Joan Edwards (Aberystwyth University) for their technical and project support. In addition, we thank Dr Yanhua Hong from the School of Electronics, Bangor University for her technical advice.

References

R. Soref,. "Mid-infrared photonics in silicon and germanium." Nature Photonics 4, no. 8, pp, 495-497, 2010

I.M. de Vargas-Sansalvador, C. Fay, T. Phelan, M. D. Fernandez-Ramos, L. F. Capitan-Vallvey, D. Diamond, and F. Benito-Lopez. "A new light emitting diode-light emitting diode portable carbon dioxide gas sensor based on an interchangeable membrane system for industrial applications." Analytica chimica acta 699, no. 2 (2011): 216-222.

S. Bayya, Shyam, J. Sanghera, W. Kim, G. Villalobos, and I. Aggarwal. "Recent advancements in multiband IR sensor windows." In SPIE OPTO, pp. 825703-825703. International Society for Optics and Photonics, 2012.

M. Casalino, G. Coppola, M. Iodice, I. Rendina, L. Sirleto "Near-Infrared Sub-Bandgap All-Silicon Photodetectors: State of the Art and Perspectives." Sensors. 2010; 10(12):10571-10600.

I.H. Campbell, and B.K. Crone. "A near infrared organic photodiode with gain at low bias voltage." Applied Physics Letters 95, no. 26 (2009): 263302-263302.

T.N. Ng, W.S. Wong, M. L. Chabinyc, S. Sambandan, and R.A. Street. "Flexible image sensor array with bulk heterojunction organic photodiode." Applied Physics Letters 92, no. 21 (2008): 213303.

C. Di, F. Zhang, and D. Zhu. "Multi-Functional Integration of Organic Field-Effect Transistors (OFETs): Advances and Perspectives." Advanced Materials 25, no. 3 (2013): 313-330.

T. Kuusela, J. Peura, B. A. Matveev, M. A. Remennyy, and N. M. Stus. "Photoacoustic gas detection using a cantilever microphone and III-V mid-IR LEDs."

Vibrational spectroscopy 51, no. 2 (2009): 289-293. 
J.S. Steckel, S. Coe-Sullivan, V. Bulović, and M. G.

Bawendi. "1.3 $\mu \mathrm{m}$ to $1.55 \mu \mathrm{m}$ tunable

electroluminescence from $\mathrm{PbSe}$ quantum dots

embedded within an organic device." Advanced

Materials 15, no. 21 (2003): 1862-1866.

J. Kettle, M. Horie, L. A. Majewski, B. R. Saunders, S.

Tuladhar, J. Nelson, and M. L. Turner. "Optimisation of

PCPDTBT solar cells using polymer synthesis with Suzuki

coupling." Solar Energy Materials and Solar Cells 95, no.

8 (2011): 2186-2193.

F. Guo, X. Z. Xiao, and J. Huang. "Fullerene

Photodetectors with a Linear Dynamic Range of $90 \mathrm{~dB}$

Enabled by a Cross-Linkable Buffer Layer." Advanced

Optical Materials 1, no. 4 (2013): 289-294.

M. Ramuz, L. Bürgi, C. Winnewisser, and P. Seitz, "High sensitivity organic photodiodes with low dark currents and increased lifetimes" Organic Electronics, no. 9(3),

(2008): 369-376.

J. Kettle, H. Waters, M. Horie, and S. W. Chang. "Effect of hole transporting layers on the performance of PCPDTBT: PCBM organic solar cells." Journal of Physics D: Applied Physics 45, no. 12 (2012): 125102.

K. Schulze, C. Uhrich, R. Schüppel, K. Leo, M. Pfeiffer, E. Brier, E. Reinold, and P. Baeuerle. "Efficient

Vacuum-Deposited Organic Solar Cells Based on a New Low-Bandgap Oligothiophene and Fullerene C60."

Advanced Materials 18, no. 21 (2006): 2872-2875.

Frederik C. Krebs, "Stability and degradation of Organic and Polymer Solar Cells," Wiley and sons, ISBN: 978-1119-95251-0, April 2012

K. W. Wong, H. L. Yip, Y. Luo, K. Y. Wong, W. M. Lau, K. H. Low, H. F. Chow, Z. Q. Gao, W. L. Yeung, and C. C. Chang. "Blocking reactions between indium-tin oxide and poly (3, 4-ethylene dioxythiophene): poly (styrene sulphonate) with a self-assembly monolayer." Applied physics letters 80, no. 15 (2002): 2788-2790.

A. Shalav, B. S. Richards, and M. A. Green. "Luminescent layers for enhanced silicon solar cell performance: Upconversion." Solar Energy Materials and Solar Cells 91.9 (2007): 829-842. 
